\title{
Short Circuit Thermally Stimulated Discharge Current Measurement on PMMA:PEMA:PVDF Ternary Blends
}

\author{
A. K. Gupta ${ }^{1,2,4^{*}}$, A. Tiwari ${ }^{1,3}$, R. Bajpai ${ }^{1}$, J. M. Keller ${ }^{1,2}$ \\ ${ }^{1}$ Department of Post Graduate Studies and Research in Physics \& Electronics, R. D. University, Jabalpur, India; \\ ${ }^{2}$ Macromolecular Research Centre, R. D. University, Jabalpur, India; ${ }^{3}$ Mata Gujari Mahila Mahavidyalaya, Jabalpur, \\ India; ${ }^{4}$ Department of Engineering Physics, Global Institute of Engineering, Patan Bypass Square, Karmeta, Jabalpur, \\ India. \\ Email: "akguptajbl@rediffmail.com
}

Received August $6^{\text {th }}, 2010$; revised December $1^{\text {st }}, 2010$; accepted June $2^{\text {nd }}, 2011$.

\begin{abstract}
The attainment of a better understanding and improvement of electrical properties of ternary blend is a task of particular scientific and economic importance. Ternary films of Poly (methylmethacrylate), Poly (ethlymathacrylate) and poly (vinlylidenefluoride) were prepared using solution cast technique. Thereafter, to study the hetro charges, homo-charges and interfacial charge formation in ternary system, the short circuit thermally stimulated discharge current (SC-TSDC) measurements were carried out on bilaterally metallized electrets. The ternary blend samples taken for the present investigations are hetrogeneous system involving three polymers differing in their conduction behaviour and dielectric property. Thus, unequal ohmic conduction currents arriving at the interface are expected to result in accumulation of charges at the interface or the Maxwell-Wagner effect. Clearly the Maxwell Wagner effect is expected to contribute discernibly to the observed TSDC's of the ternary blends. The PMMA: PEMA: PVDF: 100:100:50 blend exhibits highest tendency while 100:50:100 the least, towards the anomalous current flow. Moreover, the conductivity of 100:100:50 is found to be more and, therefore, a large amount of homocharge is injected leading to anomalous current.
\end{abstract}

Keywords: PMMA, PEMA, PVDF, Ternary Blend and SC-TSDC

\section{Introduction}

The study of electrical behavior to understand clearly the electrical conduction mechanism origin of the interfacial charge formation and charge transport behavior of ternary blended films, in order to determine the microelectronics and engineering applications for industrial use is of prime concern. As the field of polymer science continues to grow, new as well as existing techniques are being developed to study the physical properties of these materials. Most of the interesting physical properties of polymers are attributed to molecular motions which are very complex. These motions are evident by relaxations which are observed during measurements such as mechanical and dielectric measurements etc. One favorable method of assessing the physical properties of polymers is to perform short circuit thermally stimulated depolarization current (SC-TSDC) measurement. The SC-TSDC, now a well known technique [1-8], has come to be re- garded as a viable method for studying dielectric relaxation behaviour of polymers. After a conditioning phase the sample is subjected to a chosen regime of electric field and temperature changes. The result is assessed by finally heating the sample (with absence of applied electric field), usually at a linear rate and analyzing the resulting peaks of current thermograms. Originally this technique was used to measure charge detrapping in low-molecular-weight-organic and inorganic compounds. The SC-TSDC thermograms also reflect the mechanical behavior of polymers, and the resolution of SC-TSDC and dielectric data is much better than many mechanical measurements. The work reported earlier emphasizes on different interpretations for the observed results but it seems no ultimate view has yet been reached. The elucidation of the underlying charge injection and carrier migration process is vital to the ever-growing future utility of these materials. It has been shown that carrier mobility can be greatly affected by impregnating the polymers 
with suitable blending [9-12]. Thus, keeping this into account the ternary blends of Poly (methylmethacrylate) (PMMA), Poly (ethlymathacrylate) (PEMA) and poly (vinlylidenefluoride) (PVDF) were prepared and SC-TSDC characterization was carried out with different charging temperatures, charging fields and charging periods to reveal clearly the contribution of different electrical performance. However, combination of selfexisting structural-property relationship, single phase compatibility and toughness, etc. of PEMA/PMMA/ PVDF ternary blends had already been explored by Gupta et al. [13].

\section{Experimental}

\subsection{Materials}

For preparation of ternary blend specimens, commercially available polymers; poly (vinylidene fluoride) (PVDF) (Aldrich, USA) $\mathrm{M}_{\mathrm{w}} 140000$ (powder); poly (methylmethacrylate) (PMMA) (Aldrich, USA) $\mathrm{M}_{\mathrm{w}}$ 15000 (GPC); and poly (ethylmethacrylate) (PEMA) (Aldrich, USA) $\mathrm{M}_{\mathrm{w}} 34000$ (GPC) were used.

\subsection{Preparation of Ternary Films}

The solution cast technique has been utilized to prepare the ternary films of PMMA + PEMA + PVDF. Calculated quantity of the three polymers was dissolved in dimethyl formamide (DMF) their common solvent, at a temperature of $60^{\circ} \mathrm{C}$ with constant stirring. The blend films having ultimate desired concentrations of PMMA: PEMA: PVDF: 100:100:100; 50:100:100; 100:50:100; and 100:100:50, were prepared and designated as FEMA-1, FEMA-2, FEMA-3 and FEMA-4, respectively. The blends having different compositions were cast on the glass substrate in a dust free chamber by means of a spin coater (Model No. TP-1100 set) to ensure uniform ultimate thickness of 100 micron when temperature of $60^{\circ} \mathrm{C}$ was maintained. The solvent was allowed to evaporate by keeping glass substrate inside the oven for nearly 6 hours at same temperature. Thereafter, the oven was switched off and allowed to cool to room temperature $\left(20^{\circ} \mathrm{C}\right)$. The film specimen so obtained was detached from the glass substrate. The specimens obtained were in the forms of $100 \pm 0.0002$ micron thickness and $15 \mathrm{~cm}^{2}$ in size.

\subsection{Experimental Technique}

For SC-TSDC measurements the samples were bilaterally metallized over a central circular area of $5.0 \mathrm{~cm}$ diameter. The TSDC measurement consists of following stages:

1) The sample is polarized to saturation under a static electric field $\mathrm{E}_{\mathrm{p}}$, at a temperature $\mathrm{T}_{\mathrm{p}}$.
2) The sample is cooled, with the field still applied, down to a temperature $T_{0}$, (room temperature) where the dipole/charge carriers motion is hindered, i.e. where relaxation time $\tau\left(T_{0}\right)$ becomes very long (of the order of several hours) to years.

3) At $T_{0}$ the external field is removed, and a sensitive current detector is directly connected between the electrodes; because of the low temperature the dipole/charge carriers are frozen in their ordered position and the sample remains polarized even in the absence of field.

4) The sample is warmed up, usually, at a constant heating rate $\beta$ (convenient heating rates are between 1 $10 \mathrm{~K} / \mathrm{min}$ ) while the current through the detector is recorded as a function of temperature.

5) The current measurement requires a sensitive electrometer which has low detection limit of the order of $10^{-15} \mathrm{~A}$. A Keitheley Electrometer model $61^{\circ} \mathrm{C}$ was used in the present investigation.

\section{Result and Discussion}

Various results describing the behaviour of ternary blends investigated by short circuit thermally stimulated discharge current (TSDC) are discussed below.

\subsection{Temperature Dependence}

Figure 1 shows the TSDC thermogram of FEMA-1 film polarized with a field of $10 \mathrm{kV} / \mathrm{cm}$ at $40,60,80$ and $100^{\circ} \mathrm{C}$. In case of FEMA-1 samples current flows in anomalous sense for low polarizing temperatures and even for low field. Perhaps the anomalous peak appearing around $90^{\circ} \mathrm{C}$ in sample polarized at $40^{\circ} \mathrm{C}$ appears as normal peak around $80^{\circ} \mathrm{C}$ in case of sample polarized at $80^{\circ} \mathrm{C}$. On changing composition tendency towards anomalous behavior is found to become pronounced. For FEMA-2 films low field current is anomalous largely for all temperatures. As the field increases, for low tempera-

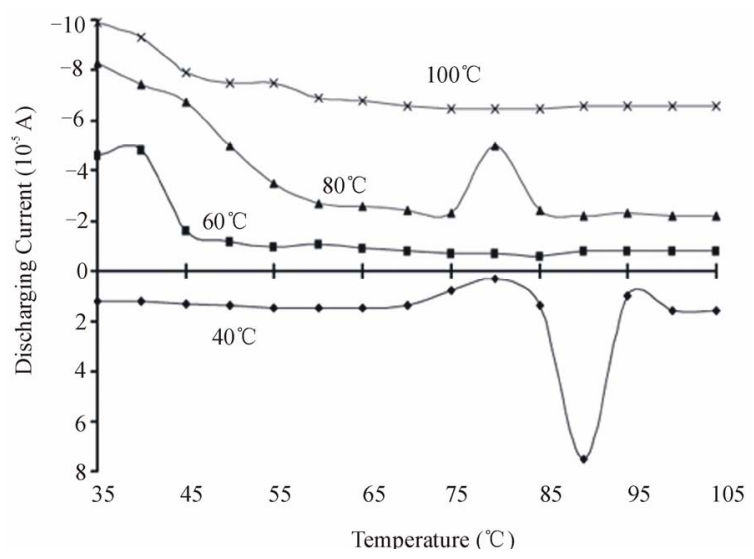

Figure 1. TSDC thermogram of PMMA: PEMA: PVDF:: 100:100:100 ternary film polarized with a field of $10 \mathrm{kV} / \mathrm{cm}$ at $40,60,80$ and $100^{\circ} \mathrm{C}$. 
tures, current is normal but for high temperature current is anomalous. At high field and high temperature the current is always anomalous, similar as for FEMA-3 and 4.

\subsection{Field Dependence}

Figure 2 shows the TSDC thermogram of PMMA: PEMA: PVDF: 100:100:100 ternary film charged at $40^{\circ} \mathrm{C}$ with $10,15,20$ and $25 \mathrm{kV} / \mathrm{cm}$ fields. In case of FEMA-1 films current flow in normal direction (except at lowest temperature) up to moderate field values. The current increases in magnitude with increase in field except for very high field for which it decreases. For FEMA-2 films current is anomalous for low polarizing temperatures and low fields and also for high temperature for all field. For moderate temperatures current is normal for all fields. The FEMA-3 films, for low temperature and low field current increase with the field. As the polarizing temperature increases, the anomalous behaviour is also observed at gradually increasing field values. Further, for FEMA-4 films current is largely anomalous for all the temperatures. Only in the case of very high field and high temperature current is normal.

\subsection{Composition Dependence}

It is evident that for the composition, specimens polarized at low temperature of $40^{\circ} \mathrm{C}$ with low field $10 \mathrm{kV} / \mathrm{cm}$ exhibits anomalous current flow, further anomalous current peak observed around $90^{\circ} \mathrm{C}$ (Figure 3). In case of FEMA-4 composition sample is considerably weakened in FEMA-3 sample composition. For samples charged at $60^{\circ} \mathrm{C}$ with $10 \mathrm{kV} / \mathrm{cm}$ only in case of FEMA-1 (Figure 4) composition anomalous current behaviour is observed astonishingly the thermograms of samples with FEMA-2 and FEMA-4, composition are exact replica of each other. From the thermograms of samples of various compositions charged at high temperature 80 and $100^{\circ} \mathrm{C}$ with low fields it is evident that FEMA-2 and FEMA-4 composi-

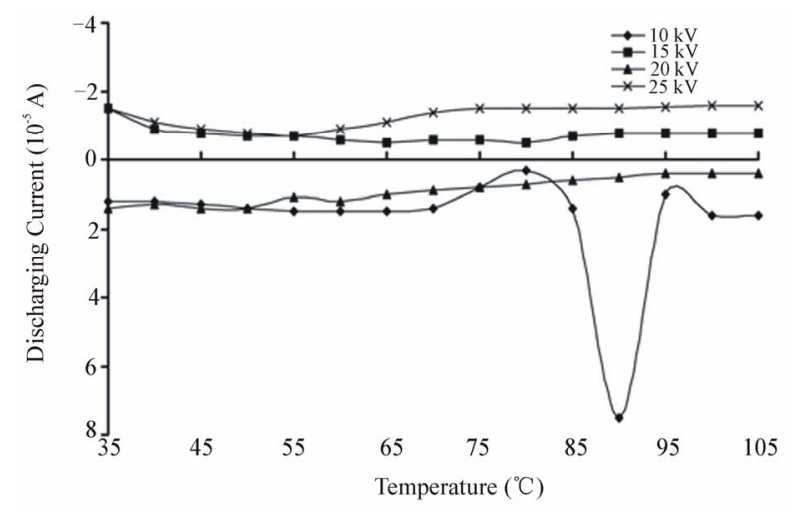

Figure 2. TSDC thermogram of PMMA: PEMA: PVDF: 100: 100: 100 ternary film charged at $40^{\circ} \mathrm{C}$ with 10, 15, 20 and $25 \mathrm{kV} / \mathrm{cm}$ fields.

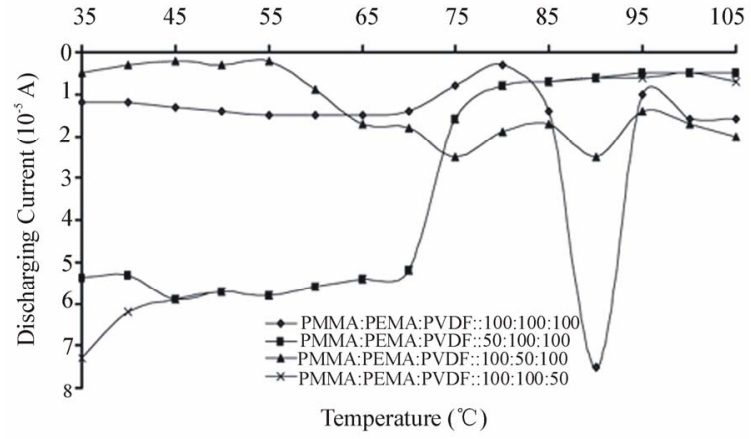

Figure 3. TSDC thermogram of ternary blends having different composition charged at $40^{\circ} \mathrm{C}$ with $10 \mathrm{kV} / \mathrm{cm}$ field.

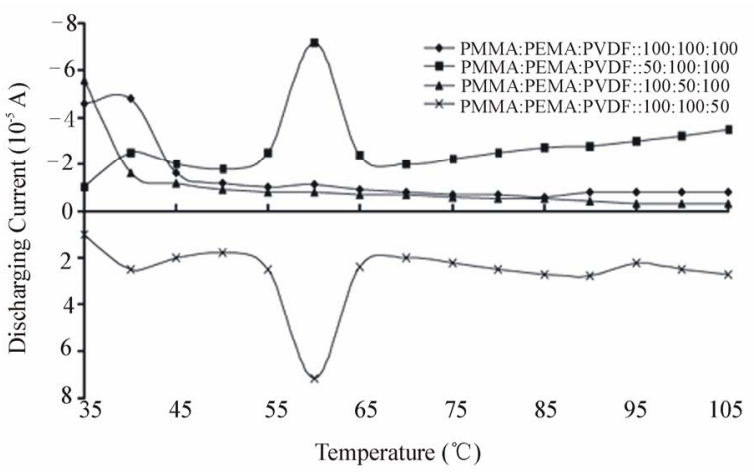

Figure 4. TSDC thermogram of ternary blends having different composition charged at $60^{\circ} \mathrm{C}$ with $10 \mathrm{kV} / \mathrm{cm}$ field.

tion samples have tendency towards anomalous current flow more pronounced as compared to other samples.

Persistent polarization in a thermally charged dielectric specimen may arise due to various mechanisms. The important among these are orientation or dipolar polarization, translational or space charge polarization and interfacial polarization. The charge originated in SCTSDC due to dipolar orientation or trapping of space charges in defect or dislocation sites is known to give rise to a uniform polarization, which is heterocharge. On the other hand, space charge build up by migration of ions over microscopic distances or accumulation near the electrodes, and interfacial or Maxwell Wagner effect gives a non uniform heterocharge, whereas trapped injected space charge results in a nonuniform homo- or hetero- charge depending upon the work function of the metal electrode.

The decay of heterocharge during SC-TSDC gives a current in a direction opposite to that of the charging current i.e., negative normal current while decay of homo charge results in a current in the same direction as the charging current called positive or anomalous current. The classical theory of Gubkin [14], Perlman [15] and others for the decay of charge in polarizing dielectrics assumes the superposition of homocharge and het- 
erocharge.

Polar polymers, in general, exhibit two main relaxations designated as $\alpha$ - and $\beta$-relaxations. The $\alpha$-relaxation arises due to the main chain segmental motion and occurs around and above $\mathrm{T}_{\mathrm{g}}$. The $\beta$-relaxation occurs in the glassy state of the polymer and is due to the hindered rotation of polar side groups around carbon-carbon link of the main chain. Sometimes $\alpha$ - and $\beta$ relaxations coalesce to give a single $\alpha, \beta$ - relaxation around $\mathrm{T}_{\mathrm{g}}$ as reported in the case of PMMA [16-18]. If there are polar side groups in the polymers side chain, capable of orienting in an electric field independent of one another and having different relaxation times, two separate $\beta$ - relaxations are observed. The relaxation reported to occur due to the space charge is designated as $\rho$ - relaxation and occurs at high temperatures. When the polymer is non-polar, it will exhibit no distinct dipolar reorientation; however, it exhibits space charge effects. Each relaxation process gives rise to a peak at its characteristic temperature during SC-TSDC.

The depolarizing current recorded in the present investigation was found to flow, in general, in the direction opposite to that of the charging current i.e. in the negative sense. Hence, processes responsible for heterocharge formation are primarily responsible for the polarization of the ternary polymeric blends. All the three polymeric components used for preparing the blend samples are polar polymers. The polymers PMMA and PEMA are branched amorphous thermoplastics. The two polymers differ only in the alkyl substituent's $\left(-\mathrm{COOCH}_{3}\right)$ and $\left(-\mathrm{COOC}_{2} \mathrm{H}_{5}\right)$ of their ester side groups. The ester side groups are polar- and so they form permanent dipoles which can be oriented during the electret formation.

It is well known that the ester side groups can rotate singly or together with the main chain segments $-\mathrm{C}-\mathrm{CH}_{2}$. Evidently their cooperative motion with the adjacent segments of the bulky main chain which may contain all together at least 10,000 monomeric links, requires more energy; hence it occurs well above the room temperature when the polymer soften and become rubbery. The polymers PMMA and PEMA, therefore exhibit $\propto$ - transitions at 103 and $66^{\circ} \mathrm{C}$ due to the disorientation of ester side groups by their cooperative motions with the adjoining segments of the main chains which at these temperatures start to rearrange their conformations.

The local motions of the polar ester side groups by rotation around their $-\mathrm{C}-\mathrm{C}$ - links with the main chain occur at much lower temperatures when the polymers are in their glassy state. Their motions are sterically hindered by the $\propto$ - methyl groups on the main chains. The polymers PMMA and PEMA, therefore, exhibit $\beta$ - relaxation in their glassy state around $-51^{\circ} \mathrm{C}$ and $-45^{\circ} \mathrm{C}$ due to release of part of their ester groups by local motions.

The third relaxation designated as $\rho$-peak has also been observed at $115^{\circ} \mathrm{C}$ for PMMA, and $85^{\circ} \mathrm{C}$ for PEMA respectively and has been ascribed to thermally stimulated space charge limited drift and diffusion of frozen excess charges.

PVDF is a semicrystalline polymer. It exhibits at least four crystalline phases $\alpha, \beta, \gamma$, and $\delta$. The $\alpha$-form is non polar while $\beta$-form is polar and is responsible for all its useful electroactive properties [19-25]. In view of the properties and relaxations of the three polymers discussed as above and the fact that the depolarization current recorded in the present investigation was found to correspond in general, to the normal current implies that the dipolar orientation processes contribute significantly to the hero charge formation. The thermograms, however, do not show characteristic dipolar peaks. Further, the TSDCs exhibit complex field and temperature dependence. This indicates that the dipole relaxations are probably masked by strong space charge effects and other phenomena. The space charges are excess charges which will migrate during the electret formation towards the electrodes. These charges may be ions or electrons and they may originate from e.g. dissociation of impurities (water, monomers, catalyst and initiation). The forming field will drive the positive charges to negative electrode and negative charges to the positive electrodes. The field motion is opposed by diffusion; moreover, during their transport parts of the charges are lost by recombination with opposite carriers. However, the field drift will dominate and charges will be piled up in the vicinity of the electrodes.

In hetero- electrets the excess charges are intrinsic and bipolar. They originate from those charges that first took part in the conduction and were next accumulated near the electrodes during the formation. The occurrence of space charge polarization requires that there be enough carriers of a sufficiently high mobility and this condition is satisfied only if the conduction is reasonably high. Hence, space charge formation occurs at high temperature close to and above $\mathrm{T}_{\mathrm{g}}$. The excess charges may also be injected from the electrodes depending upon the work function of the electrode material and the polymer. Under the condition of high temperature, when the mobility of charge carrier is high these charges may be localized in various traps existing in the polymer. During the depolarization cycle the frozen in localized excess charges are thermally mobilized and they start moving under their own field towards the shorted electrodes and also by diffusion generating hetro current.

Incidentally in hetrogeneous hetro-electrets of partially 
crystalline polymers the intrinsic excess charges will mainly pile up at the phase boundaries. They are supplied there by unequal omic conduction currents within the two components or phases (interfacial or Maxwell Wagner charging). The ternary blend samples taken for the present investigations are hetrogeneous system involving three polymers differing in their conduction behaviour and dielectric properties. Thus, unequal ohmic conduction currents arriving at the interface are expected to result in accumulation of charges at the interface or the Maxwell-Wagner effect. Since the ohmic conductivities are thermally activated, the Maxwell-Wagner effect produces permanent polarization when the sample is exposed to field-temperature cycle. During SC-TSDC the accumulated charge is neutralized by new carriers of opposite polarity that are conveyed to the interface by conduction currents which are created by the interfacial charge itself. Clearly the Maxwell Wagner effect is expected to contribute discernibly to the observed SCTSDC of the ternary blends.

It has been observed that the depolarization current in many cases is found to flow in the same direction as the charging current. The depolarization current flowing in the same direction as charging current is called positive current or anomalous current while the depolarizing current flowing in a direction opposite to the charging current is called negative and normal current. Particularly for samples charged at low temperatures or at high temperatures with high field, the current exhibits the anomalous behavior. Since the direction of the current is opposite to that expected from the depolarization of dipoles, it is likely to be due to electrode polarization or injected homo space charge.

The anomalous behaviour of SC-TSDC current may be understood in terms of localization of excess charges in various available traps in the bulk of the polymers. At low temperature when the mobility of the charge carriers is low, the charge is largely localized in shallow traps. However, at high temperature, the charge carriers with high mobility are shifted to deeper traps. The number of such trapped charge carriers increases with the increase in the value of applied step field. It appears that owing to the polar character of the three polymers, excess charge carriers in sufficiently high concentration exist in the ternary blend samples. With limited mobility at low polarizing temperature and under the directing action of the field, these carriers in large number occupy the shallow traps only in the bulk of the polymer. The releases of a very large number of such charge carriers during the depolarization cycle may exceed the charge exchange rate of the charging electrode resulting in the blocking of the electrode and a consequent net carriers back flow towards the near electrode. This gives rise to anomalous

\section{SC-TSDC current.}

As the temperature increases, mobility of the charge carriers also increases; consequently the excess charge is shifted to deeper traps. The release of charge carriers from such traps requires more energy. Thus, release of trapped charge carriers from deeper traps in limited number may not be sufficient enough so as to cause blocking of the charging electrode. Hence, carriers flow towards the charging electrode resulting in normal current in negative direction as has been observed from samples polarized with moderate fields at moderate temperatures. For high polarizing temperature, charge carriers, with high mobility in large numbers under directing action of high polarizing field occupy shallow traps as well as deep traps. The release of trapped charge carriers during TSD cycle in very large number may, therefore, again surpass the carrier exchange rate of the electrode causing its blocking and hence a net carrier flow towards rear electrode giving positive anomalous current.

The anomalous current may also result from the injected charge carriers. The anomalous current in terms of injected homo space charges can be understood as follows. Considering one type of carrier, i.e. electrons for simplicity, we may assume a distribution of injected space charges just after charging such that its density $\mathrm{n}$ (x, t) drops with increasing distance $X$ from the injecting electrode. Thus, the field $\mathrm{F}(0, \mathrm{t})$ at the injecting electrode at any instant of time is greater than the field at any other point of the dielectric $(\mathrm{F}(0, \mathrm{t})>\mathrm{F}[\mathrm{d}, \mathrm{t})]$, and there exists a zero field plane at a distance say $\mathrm{X}_{0}$ towards the right. The appearance of anomalous current requires the suppression of the carriers to the left which results in a net carrier flow to the right and the movement of zero field plane $\mathrm{X}_{0}$ to the right.

As the carrier mobility increases with temperature and the density of injected space charge increases with the applied field, a strong space charge is expected near the injecting electrode at high charging field and high temperature. Such a situation is expected to result in a high return rate of carriers to the injecting electrode. As a result, the return rate of the carriers may surpass the charge exchange rate of the electrode leading to the blocking of the electrode. This blocking suppresses the carriers flow to the left (injecting) electrode and may result in the movement of $\mathrm{X}_{0}$ towards right electrode causing anomalous current. Figures 5 (a) and (b), shows the distribution of injected space charge and internal field.

The injected homo space charge may also produce a non-uniform distribution of space charges. The injected space charges may be localized in various trapping sites available in the specimen. Under certain conditions of charging field and temperature the positive charges injected from the anode may migrate under the action of the 

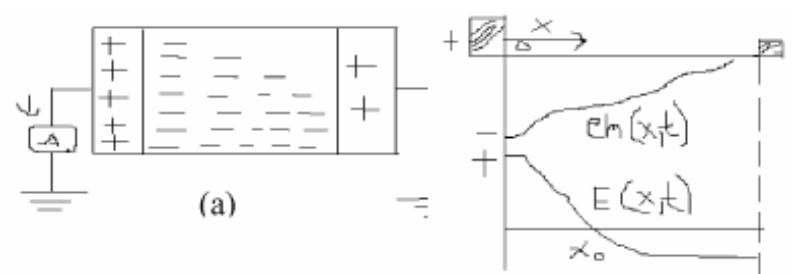

(b)

Figure 5. (a) and (b) distribution of injected space charge and internal field.

internal field and pile up in front of the cathode and may become immobilized on cooling during electrets formation. On removing the field and heating in short circuit, this positive charge is released and the net flow will be positive due to its accumulation in the vicinity of the cathode. Similarly, the injected negative charges piled up in front of the anode may flow towards the anode. The flow of positive charges towards the cathode and negative charges towards the anode will lead to anomalous current or homo charge flow.

From the various thermograms it is clear that the tendency towards anomalous current flow depends on the ternary blend composition. The FEMA-4 film exhibits highest tendency while FEMA-3 the least, towards the anomalous current flow. In case of FEMA-4 film the current exhibits normal behavior only for highest forming temperature and field. Thus, change in the $\mathrm{wt} \%$ composition of the ternary blend significantly modifies the chemical structure and hence trap structure of the blend.

It appears that reduction in wt $\%$ of PVDF in the blends modifies the structure such that there results an increase in the number of migration states or shallow traps. The detrapping of charge carriers from these shallow states in large number leads to blocking of near electrode and consequently there results a net carriers flow towards farther electrode. In case of FEMA-3 samples reduction in the bulky ethyl group leads to increase in the density of ester group and trapping sites. Detrapping of charge carriers from these traps requires higher activation energy; this leads to decrease in the number of released charge carriers not exceeding the charge carrier exchange rate of the electrode, and hence to a normal current.

Increase in the tendency of depolarization current towards anomalous behavior may also be understood in terms of injected homocharge. It appears that the conductivity of FEMA-4 is maximum so that a large amount of homo charge is injected. PVDF is a semicrystalline polymer while PMMA and PEMA both are amorphous. Thus, in FEMA-4 blends amorphous content is relatively more. We know that the conductivity of amorphous part is more as compared to crystalline part. Hence, conduc- tivity of FEMA-4 being more and a large amount of homocharge is injected leading to anomalous current.

From the SC-TSDC thermograms of ternary blends polarized with the field of $10 \mathrm{kV} / \mathrm{cm}$ at temperature $60^{\circ} \mathrm{C}$ (Figure 4), it is evident that thermograms of FEMA-2 and FEMA-4 films are exactly replica of each other. The FEMA-2 blend exhibits normal current with a hump and a well developed peak around temperatures 40 and $60^{\circ} \mathrm{C}$, respectively. On the contrary FEMA-4 film shows anomalous current with hump and peak around the same temperatures. Polymer PVDF is known to act as plasticizer for PMMA and PEMA. Further, substituent ethyl group is larger as compared to methyl group. The larger ethyl group of PEMA which participates in the orientation of ester group of methacrylic polymer will push the main chain in the polymer farther apart thereby causing internal plasticization. Thus, the highly plasticized ternary blend FEMA-2 exhibits increased mobility of the main chain and the release of the dipolar ester group by their cooperative motion with the adjoining segments of the main chains is manifested in the form of hump and peak in the normal direction.

It may also be inferred here that the hump and peak appearing around 40 and $60^{\circ} \mathrm{C}$ are actually the manifestation of dipolar relaxations associated with $\alpha$-transitions observed around glass transition temperatures of PEMA and PMMA or $\beta$-relaxation associated with the chain fold motion in the amorphous region of PVDF. The plasticization is known to the lower the $T_{g}$, and it appears that $\mathrm{T}_{\mathrm{g}}$ 's of the PEMA and PMMA are shifted to around 40 and $60^{\circ} \mathrm{C}$ [13]. Reduction in $w t \%$ of PVDF results in a less plasticized ternary blend or conversely a relatively hardened blend material. The hardening results in an imperfect contact between electrode and the sample. This increases chances of breakdown in the electrode samples interface and consequently enhances homocharging of the sample. In the shorted homo-electrets the excess injected homo charge localized in migration states and deep traps will moves back to the near electrode on the injection side. These homo-charge motions thus attract image charges to this electrode rather than liberating them. The resultant current is the net homo current and the hump and peaks current are in fact the result of opposed dipolar and excess charge drift. Since the mobility of the extrinsic carriers depends on the temperature and free volume in a way similar to that of the chain segments of the polymer. Increased homocharge injection may also be understood in terms if increased conductivity of FEMA-4 film. Reduction in the $\mathrm{wt} \%$ content of semicrystalline polymer PVDF entails decrease in the net crystalline component and consequently an increase in the net amorphous content in the ternary blends. Since the conductivity of amorphous polymer is more so that in 
this blend there is more injection of homo charge from the electrode into the polymeric blend. Further, as said earlier increase in the net amorphous content probably is accompanied by increase in the density of migration states (shallow traps) in which a large amount of excess charge is localized. The delocalization and release of a large amount of charge from these states results in anomalous SC-TSDC in FEMA-4.

\section{Conclusions}

The SC-TSDC measurement helps to understand the hetrogeneous system involved in the three polymers which are differ in their conduction behaviour and dielectric properties. The SC-TSDC measurement shows the unequal ohmic conduction currents arriving at the interface, which are expected to result in accumulation of charges at the interface or the Maxwell-Wagner effect. Thus Maxwell Wagner effect clearly expected to contribute discernibly to the observed TSDC's of the ternary blends and hence making it useful material for microelectronics and much special purpose insulation.

\section{REFERENCES}

[1] T. Fahmy and M. T. Ahmed, "Thermal Induced Structural Change Investigations in PVC/PEMA Polymer Blend," Polymer Testing, Vol. 20, No. 5, 2001, pp. 477-484. doi:10.1016/S0142-9418(00)00060-X

[2] J. Vanderschueren and J. Gasiot, "Topics in Applied Physics: Thermally Stimulated Relaxations in Solids," Springer Verlag, Berlin, 1979.

[3] C. Viorel, M. M. Doina, R. Constantin, I. Monica, M. Yann and D. Florea, "Mesomorphic Behaviour and TSDC Measurements of Ortho-Metallated Palladium(Ii) and Platinum(Ii) Complexes with S,O-Donor Co-Ligands," Liquid Crystals, Vol. 36, No. 9, 2009, pp. 123-132. doi:10.1080/02678290802696173

[4] S. Classen, M. Vogt and J. H. Wendorff, "Phase Behaviour and Structure Formation in Ternary Blends: Studies on Blends of PVDF/ PMMA/ PVAC," Polymer Advance Technology, Vol. 6, 2003, pp. 616-625. doi:10.1002/pat.1995.220060905

[5] D. H. F. Kanda, H. N. Nagashima, J. A. Malmonge, W. K. Sakamoto and G. O. Chierice, "Effect of Polyol Excess on the Electrical Property of Vegetable-Polyurethane Film," Journal Material Science, Vol. 43, 2008, pp. 5436-5440. doi:10.1007/s10853-008-2843-3

[6] T. Hirayama, A. T. Takahashi and K. Koyama, "Thermally Stimulated Current Measurements for a Blend of Poly (Vinylidene Fluoride-Triflouroethiene) Copolymer and Poly (Methyl Methacrylate)," Japan Journal Polymer Science Technology, Vol. 61, 2004, pp. 335-340.

[7] M. T. Ahmed, "Thermally Stimulated Discharge Current and Thermal Sampling Studies on Poly (Vinyl Chloride-Co-Vinylacetate-Co-2-Hydroxypropylacrylate)/Poly
(Methyl Methacrylate) Blends," International Journal of Materials Sciences, Vol. 4, 2009, pp. 12-18.

[8] P. Saxena, P, M. S. Gaur and P. K. Khare, "Effect of Blending with Polysulfone on Thermally Stimulated Discharge Behavior of Polyvinylidenefluoride Films," Engineering, Vol. 48, 2009, pp. 415-422.

[9] Y. Yin, Z. Li, X. Li and P. Jiang, "Thermally Stimulated Currents of $\mathrm{SiO}_{2} /$ Low-Density Polyethylene," Micro and Nanocomposite, Vol. 5, 2010, pp. 385-390.

[10] T. Mitra, J. M. Keller and R. B. Bajpai, "Thermally Stimulated Depolarization Current Investigation of Poly (Phenylene Oxide): Poly (Styrene) Polymer Blends," Indian Journal of Physics, Vol. 79, 2005, pp. 361-366.

[11] V. S. Sangawar, R. J. Dhokne, A. U. Ubale, P. S. Chikhalikar and S. D. Meshram, "Structural Characterization and Thermally Stimulated Discharge Conductivity (Tsdc) Study in Polymer Thin Films," Bulletin Material Science, Vol. 30, No. 2, 2007, pp. 163-166. doi: 10.1007/s12034-007-0029-2

[12] R. A. Singh, R. K. Gupta and S. K. Singh, "Preparation and Characterization of Polymer Composite Based on Charge Transfer Complex of Phenothiazine-Iodine in Polystyrene," Bulletin Material Science, Vol. 28, No. 5, 2005, pp. 423-429. doi:10.1007/BF02711231

[13] A. Tiwari, A. K. Gupta, R. Bajpai and J. M. Keller, "Structural and Mechanical Study of In-Situ Generated Poly (Methylmethacrylate) (PMMA), Poly (Ethlymathacrylate) (PEMA) and Poly (Vinlylidenefluoride) (PVDF) Ternary Films," Polymer Plastic Technology Engineering, Vol. 49, 2010, pp. 573-580. doi: $10.1080 / 03602551003652722$

[14] A. N. Gubkin, "Electrets," Academy of Sciences, Moscow, 1961 .

[15] M. M. Perlman and S. Unger, "TSC Study of Traps in Electron-Irradiated Teflon and Polyethylene," Journal Physics D Applied Physics, Vol. 5, No. 11, 1972, pp. 2115-2119. doi:10.1088/0022-3727/5/11/319

[16] T. Heijiboer, "Mechanical properties and Molecular Structure in Physics of Non Crystalline Solids," North Holand Publisher, Amsterdam, 1965.

[17] J. M. Reboul, "Simultaneous Space Charge and TSDC Measurements on Polarized," Polyme, Vol. 13, 2006, pp. 1016-1022.

[18] C. Choure, J. M. Keller and R. Bajpai, "Thermally Stimulated Depolarization Current Behavior of Polyethylene/Polyvinlylacetate Blends Effect of Blending," Journal Applied Polymer Science, Vol. 101, 2006, pp. 30403045. doi:10.1002/app.23849

[19] V. Turnhout, "Thermally Stimulated Discharge of Polymer Electrets," Elsevier, Amsterdam, 1975.

[20] N. G. Mccrum, R. E. Read and G. Williams, "An Electric and Dielectric Effects in Polymeric Solids," Wiley, New York, 1967.

[21] V. V. R. R. Narasimha and A. Kalpalatha, "Thermally Stimulated Discharge Currents in Polyvinyl Pyrrolidone Polymer Films," Material Chemistry Physics, Vol. 17, No. 
4, 1987, pp. 317-324. doi:10.1016/0254-0584(87)90084-8

[22] M. Madani, N. A. Maziad and R. M. Khafagy, "Thermally Stimulated Depolarization Current and Thermal Analysis Studies of Gamma Irradiated Lithium-Salt/Polymer Electrolyte Blends," Journal Macromolecular Science B, Vol. 46, No. 6, 2007, pp. 1191-1203. doi: $10.1080 / 00222340701629224$

[23] R. A. Creswell, M. M. Perlman and M. A. Kabayama,
"Dielectric Properties of Solids," Plenum, New York, 1972.

[24] A. J. Lovinger, "Development in Crystalline Polymers-1," Applied Science, London, 1982.

[25] M. Borisova, O. Galukov and A. Kanapitsas, "Polarization Phenomena in Blends of Polyurethane and Styrene-Acrylonitrile," Electrets, ISE 10 Proceedings, 1999, pp. 573-576. 